\title{
Non-Abelian Quantum Transport and Thermosqueezing Effects
}

\author{
Gonzalo Manzano $\odot,{ }^{1,2, *}$ Juan M.R. Parrondo, ${ }^{3}$ and Gabriel T. Landi ${ }^{4}$ \\ ${ }^{1}$ Institute for Cross-Disciplinary Physics and Complex Systems (IFISC) UIB-CSIC, Campus Universitat Illes \\ Balears, Palma de Mallorca E-07122, Spain \\ ${ }^{2}$ Institute for Quantum Optics and Quantum Information (IQOQI), Austrian Academy of Sciences, \\ Boltzmanngasse 3, Vienna 1090, Austria \\ ${ }^{3}$ Departamento de Estructura de la Materia, Física Térmica y Electrónica and GISC, Universidad Complutense \\ Madrid, Madrid E-28040, Spain \\ ${ }^{4}$ Instituto de Física da Universidade de São Paulo, São Paulo 05314-970, Brazil
}

(Received 13 November 2020; revised 16 March 2021; accepted 6 December 2021; published 6 January 2022)

\begin{abstract}
Modern quantum experiments provide examples of transport with noncommuting quantities, offering a tool to understand the interplay between thermal and quantum effects. Here we set forth a theory for nonAbelian transport in the linear response regime. Our key insight is to use generalized Gibbs ensembles with noncommuting charges as the basic building blocks and strict charge-preserving unitaries in a collisional setup. The linear response framework is then built using a collisional model between two reservoirs. We show that the transport coefficients obey Onsager reciprocity. Moreover, we find that quantum coherence, associated with the noncommutativity, acts so as to reduce the net entropy production, when compared to the case of commuting transport. This therefore provides a clear connection between quantum coherent transport and dissipation. As an example, we study heat and squeezing fluxes in bosonic systems, characterizing a set of thermosqueezing coefficients with potential applications in metrology and heat-to-work conversion in the quantum regime.
\end{abstract}

DOI: 10.1103/PRXQuantum.3.010304

\section{INTRODUCTION}

In the simplest scenario, putting in contact two thermal systems at different temperatures causes them to exchange heat [1]. The amount of heat exchanged is proportional to the temperature gradient, which is known as Fourier's law. Similarly, a gradient of chemical concentration (or electric voltage) generates a flow of particles (or electrons), as predicted by Fick's (Ohm's) law. There are also cross effects: a gradient of temperature causes a flow of particles (the Seebeck effect), while a gradient of chemical potential causes a flow of heat (Peltier effect). These phenomena form the basis of a great variety of applications in thermoelectricity, thermomagnetism, and galvanomagnetic phenomena [2,3]. One of the first major advances in the construction of modern nonequilibrium thermodynamics was the development of a solid theoretical basis

*gmanzano@ucm.es

Published by the American Physical Society under the terms of the Creative Commons Attribution 4.0 International license. Further distribution of this work must maintain attribution to the author(s) and the published article's title, journal citation, and DOI. for explaining them, as provided by Onsager's reciprocity theory $[4,5]$.

Heat and particle transport deals with observables that commute at the quantum level. This is what we will refer to as Abelian transport and is known to crucially impact the thermodynamics of both classical [6,7] and quantum systems [8-13]. Non-Abelian transport, on the other hand, is associated with observables that do not commute, owing to the appearance of other types of quantum excitations [14]. Examples include the transport of energy and magnetization in certain types of spin chains [15], such as the transverse-field Ising model $[16,17]$; transport of energy and particles in the Kitaev model of superconducting wires [18]; and transport of energy and bosonic squeezing in quantum optical systems $[19,20]$.

The fundamental implications of noncommuting conserved quantities on the thermodynamics of quantum systems has only started to be explored [11,21-24] and several questions remain open. Filling this gap would be both valuable and timely, for at least two reasons. First, the phenomenon is already well within reach of several modern quantum experimental platforms, such as ultracold atoms [25,26] or optomechanical devices [27,28]. Second, non-Abelian transport provides a perfect arena for understanding the interplay between thermal phenomena and 
quantum coherence, a critical issue that has been at the center of many quantum thermodynamic studies in the last years [29-39]. Since exchanges of extra conserved quantities may contribute to both heat and work, non-Abelian transport theory may help to clarify the thermodynamics of squeezing-powered engines [40-43] and other nonthermal devices [44-46].

In this paper we establish a framework for non-Abelian transport in the linear response regime, generalizing Onsager's reciprocity theory to the case of arbitrary noncommuting charges. Noncommutativity breaks joint fluctuation theorems, which are often used to derive Onsager reciprocity [47]. It also leads to a disconnection between different notions of equilibrium, associated with the maximum entropy principle and complete passivity $[21,22]$. To overcome these difficulties, we introduce a collisional model, which allows us to formulate non-Abelian transport in terms of the notion of charge preservation.

Our results show that the transport coefficients, as well as the entropy production, can be written in terms of the so-called $y$ covariances [48-52], which provide a generalization of the notion of covariances to noncommuting observables. This is further expressed in terms of the Wigner-Yanase-Dyson skew information [49], a quantifier of quantum coherence. Inspired by Refs. [50,51], we then use this to pinpoint the role of coherence in the resulting transport properties. More specifically, we find that quantum coherence, associated with the noncommutativity of the thermodynamic charges, acts so as to reduce the total entropy production of the process. As an application, we study the transport of heat and squeezing in bosonic systems. Our framework allows the introduction and characterization of the squeezing version of the Seebeck and Peltier effects. The interesting avenues of research this opens are exemplified by metrology in a squeezing-based thermocouple, and heat-to-work conversion in an autonomous engine.

\section{FORMAL FRAMEWORK}

Let $\left\{Q_{k}\right\}$ denote a set of not necessarily commuting observables, which we henceforth refer to as thermodynamic charges (e.g., the Hamiltonian $H$ and number of particles operators $N$ in the Abelian case). We assume that each charge is associated with a corresponding affinity $\lambda_{k}$, as described by the generalized Gibbs ensemble (GGE) [53]

$$
\pi_{\lambda}=\frac{e^{-\sum_{k} \lambda_{k} Q_{k}}}{Z}
$$

where $Z=\operatorname{Tr}\left[e^{-\sum_{k} \lambda_{k} Q_{k}}\right]$ is the partition function. GGEs can be viewed as the natural generalization of the grandcanonical ensemble, $e^{-\beta(H-\mu N)} / Z$, where $\beta=1 / T$ is the inverse temperature and $\mu$ the chemical potential (the affinities of $H$ and $N$ are thus $1 / T$ and $\mu / T$ ). These states are known to play a fundamental role in describing the relaxation of integrable many-body quantum systems after a sudden quench [54,55].

We consider non-Abelian transport between two reservoirs, 1 and 2, each described by their own set of charges $\left\{Q_{k}{ }^{(i)}\right\}$ and prepared in a GGE of the form (1), but with possibly different affinities $\lambda^{(1)}=\left(\lambda_{1}^{(1)}, \lambda_{2}^{(1)}, \ldots\right)$ and $\lambda^{(2)}=$ $\left(\lambda_{1}^{(2)}, \lambda_{1}^{(2)}, \ldots\right)$. Each reservoir consists of an infinite pool of simple subsystems or units. To model the interaction between the two reservoirs, we use a collisional model approach that simplifies the problem and has demonstrated to be very useful in the theory of open quantum systems [56-60]. In its most basic version, a small system interacts sequentially (or "collides") with fresh units coming from a reservoir. Even though the evolution in each interaction is unitary, tracing the reservoir units after interaction leads to dissipative dynamics on the system ruled (under mild conditions) by the same Markovian master equation as in typical weak-coupling perturbation scenarios [61]. As in Ref. [59], here we extend this collisional approach to the problem of transport, that is, the two reservoirs interact with each other through an infinite sequence of small and equivalent collisions as sketched in Fig. 1.

In each collision two arbitrary subsystems, 1 and 2, in states $\pi_{\lambda^{(1)}}^{(1)}$ and $\pi_{\lambda^{(2)}}^{(2)}$ interact unitarily according to the map

$$
\rho_{12}^{\prime}=U\left(\pi_{\lambda^{(1)}}^{(1)} \otimes \pi_{\lambda^{(2)}}^{(2)}\right) U^{\dagger},
$$

where $U$ is a unitary operator [62]. In order to enforce transport during the interaction, we assume that $U$ is charge preserving; that is, it satisfies [63-65]

$$
\left[U, Q_{k}^{(1)}+Q_{k}^{(2)}\right]=0 \quad \text { for all } k .
$$

This implies that any increase of a charge in subsystem $1, Q_{k}^{(1)}$, will always be associated with an equal decrease

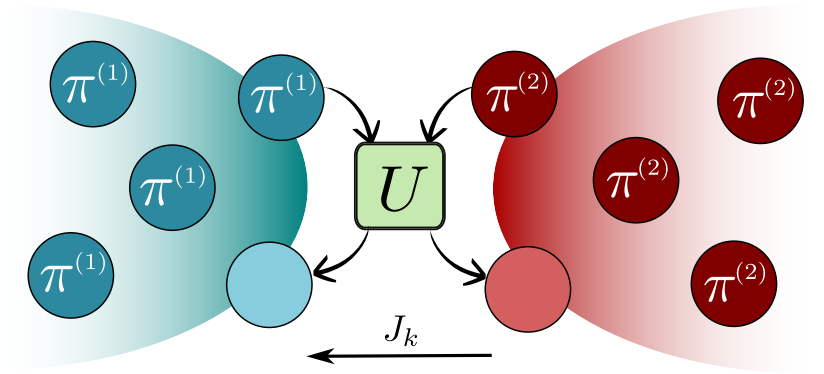

FIG. 1. Collisional transport framework between two reservoirs (red and blue regions). At each time step two subsystems prepared in GGE states $\pi^{(1)}$ and $\pi^{(2)}$ interact through a chargepreserving unitary operation $U$ (green box). After interacting, the two subsystems are returned to their respective reservoirs, producing a net flow of charges $J_{k}$. 
of the same charge in subsystem $2, Q_{k}^{(2)}$. This property is crucial in order to have proper transport; it would make no sense to talk about a quantity being "transported," if it could be spontaneously created or destroyed. When Eq. (3) is satisfied, one may unambiguously define the current associated with the charge $Q_{k}$ per collision as

$$
J_{k}:=\left\langle Q_{k}^{(1)}\right\rangle_{f}-\left\langle Q_{k}^{(1)}\right\rangle_{i}=-\left\langle Q_{k}^{(2)}\right\rangle_{f}+\left\langle Q_{k}^{(2)}\right\rangle_{i},
$$

where $\langle\cdot\rangle_{f}$ and $\langle\cdot\rangle_{i}$ refer to expectation values in the final and initial states of map (2), respectively. Equation (3) implies that the fixed point of map (2) is always the global equilibrium state $\pi:=\pi_{\lambda}^{(1)} \otimes \pi_{\lambda}^{(2)}$, where both systems have the same affinities. As a consequence, the currents will only flow provided there is a gradient of affinities.

The net entropy produced per collision can then be written in the usual form, as a product of fluxes times forces as $[1,10]$

$$
\Sigma=\sum_{k} \delta \lambda_{k} J_{k} \geq 0
$$

where $\delta \lambda_{k}=\lambda_{k}^{(1)}-\lambda_{k}^{(2)}$. The positivity of $\Sigma$ can be made manifest by expressing it in terms of information theoretic quantities [33,66-68] (see Appendix A for details).

\section{ONSAGER COEFFICIENTS}

We are interested in the linear response regime, which follows by setting $\lambda_{k}^{(2)}=\lambda_{k}$ and $\lambda_{k}^{(1)}=\lambda_{k}+\delta \lambda_{k}$, where $\delta \lambda_{k}$ is assumed to be small. In the standard paradigm, the currents $J_{k}$ respond linearly to the affinity differences $\delta \lambda_{k}$ to leading order, namely,

$$
J_{k}=\sum_{\ell} L_{k \ell} \delta \lambda_{\ell}
$$

where $L_{k \ell}$ represent the transport coefficients of the model and form the so-called Onsager matrix $\mathbb{L}$ [4]. The entropy production in Eq. (5) then becomes a quadratic form,

$$
\Sigma=\sum_{k, l} L_{k, l} \delta \lambda_{k} \delta \lambda_{l}=\delta \boldsymbol{\lambda} \mathbb{L} \boldsymbol{\delta} \lambda^{T}
$$

with $\delta \lambda=\left(\delta \lambda_{1}, \delta \lambda_{2}, \ldots\right)$. The exact form of $\mathbb{L}$ can be found from the underlying dynamics. The fact that the charges are noncommuting, however, introduces fundamental modifications to the usual linear response treatments. Notwithstanding, it turns out that $L_{k \ell}$ can be expressed in a convenient, and particularly illuminating form, as summarized in the following theorem.

Theorem 1: Provided that the set of charges $\left\{Q_{k}\right\}$, as well as the dynamics, are time-reversal invariant, the Onsager coefficients $L_{k \ell}$ associated with map (2) can be written as

$$
L_{k \ell}=\frac{1}{2} \int_{0}^{1} d y \operatorname{cov}_{y}\left(\tilde{Q}_{k}^{(1)}-Q_{k}^{(1)}, \tilde{Q}_{\ell}^{(1)}-Q_{\ell}^{(1)}\right),
$$

where $\tilde{Q}_{k}^{(1)}:=U^{\dagger} Q_{k}^{(1)} U$ and

$$
\operatorname{cov}_{y}(A, B)=\operatorname{Tr}\left(A \pi^{y} B \pi^{1-y}\right)-\operatorname{Tr}(A \pi) \operatorname{Tr}(B \pi)
$$

is the so-called "y covariance" $[48,49]$ evaluated in the global equilibrium state $\pi=\pi_{\lambda}^{(1)} \otimes \pi_{\lambda}^{(2)}$.

The proof of the theorem can be found in Appendix B. It follows from the expansion of the initial state of system 1 , to leading order in $\delta \lambda_{k}$, as

$$
\pi_{\lambda^{(1)}}^{(1)} \simeq \pi_{\lambda}^{(1)}+\left.\sum_{\ell} \delta \lambda_{\ell} \frac{\partial \pi_{\lambda+\delta \lambda}^{(1)}}{\partial\left(\delta \lambda_{\ell}\right)}\right|_{\delta \lambda_{\ell}=0} .
$$

Substituting this expansion into the current expressions $J_{k}=\left\langle Q_{k}^{(1)}\right\rangle_{f}-\left\langle Q_{k}^{(1)}\right\rangle_{i}$, and using the global map (2), allows us to identify the Onsager coefficients as

$$
L_{k \ell}=\operatorname{Tr}\left[\left(\tilde{Q}_{k}^{(1)}-Q_{k}^{(1)}\right)\left(\left.\frac{\partial \pi_{\lambda+\delta \lambda}^{(1)}}{\partial\left(\delta \lambda_{\ell}\right)}\right|_{\delta \lambda_{\ell}=0} \otimes \pi_{\lambda}^{(2)}\right)\right],
$$

where $\tilde{Q}_{k}^{(1)} \equiv U^{\dagger} Q_{k}^{(1)} U$. Rewriting this expression by using Feynman integral representations we are able to obtain two equivalent expressions for the Onsager coefficients $L_{k \ell}$ from which, by assuming time-reversal invariant currents, the form in Eq. (8) is obtained. The case of non-timereversal invariant systems is treated in Appendix C.

The $y$ covariance in Eq. (9), often used in condensed matter [48], provides a generalization of the notion of covariance to noncommuting observables. In fact, if the process is Abelian, Eq. (8) reduces to $L_{k \ell}=\frac{1}{2} \operatorname{cov}\left(\tilde{Q}_{k}^{(1)}-\right.$ $\left.Q_{k}^{(1)}, \tilde{Q}_{\ell}^{(1)}-Q_{\ell}^{(1)}\right)$. But in the non-Abelian case, no such simplification exists.

Corollary 1: Using the expression for the Onsager coefficients in Eq. (8), the entropy production rate per collision in Eq. (5) can be written as

$$
\Sigma=\frac{1}{2} \int_{0}^{1} d y \operatorname{cov}_{y}(D, D)
$$

where we defined the operator $D:=\sum_{k} \delta \lambda_{k}\left(\tilde{Q}_{k}^{(1)}-Q_{k}^{(1)}\right)$.

Let us now explore the main consequences of Theorem 1 and Corollary 1. First, noting that $\operatorname{cov}_{y}(A, A) \geq 0$ for any operator $A$ [49], we recover the positivity of the entropy production in the form (11). Since $\Sigma \geq 0$ for any set of 
gradients $\left\{\delta \lambda_{k}\right\}$, it then follows that $\mathbb{L}$ is a positive semidefinite matrix. Second, and more importantly, using the fact that $\operatorname{cov}_{y}(A, B)=\operatorname{cov}_{1-y}(B, A)$, it follows that the Onsager coefficients are symmetric, that is, $L_{k \ell}=L_{\ell k}$ for all $k, \ell$, even if the charges do not commute. This is therefore the non-Abelian generalization of Onsager's reciprocity relations. If the charges are not time-reversal invariant, as is the case, for instance, of spin chains, the matrix $\mathbb{L}$ is not necessarily symmetric, but we instead recover Onsager-Casimir reciprocity [69] (see Appendix C for details).

Next we exploit the properties of the $y$ covariance to split the entropy production rate in Eq. (11) in classical and quantum contributions. For this purpose, we make use of the general relation $\operatorname{cov}_{y}(A, A)=\operatorname{var}(A)-I_{y}(\pi, A)$, where $\operatorname{var}(A)=\left\langle A^{2}\right\rangle_{\pi}-\langle A\rangle_{\pi}^{2}$ is the conventional variance, and $I_{y}(\pi, A)$ is the so-called Wigner-Yanase-Dyson (WYD) skew information $[49,50]$, defined as

$$
I_{y}(\pi, A)=-\frac{1}{2} \operatorname{Tr}\left(\left[\pi^{y}, A\right]\left[\pi^{1-y}, A\right]\right) .
$$

This quantity measures the amount of quantum coherence that $A$ has in the eigenbasis of $\pi$ (or, alternatively, the coherence of $\pi$ in the $A$ eigenbasis) [70]. It is always nonnegative and becomes zero if and only if $A$ commutes with $\pi$. Substituting this into the entropy production expression in Eq. (11), we find that

$$
\Sigma=\frac{1}{2} \operatorname{var}(D)-\frac{1}{2} \int_{0}^{1} d y I_{y}(\pi, D) .
$$

While for Abelian transport only the first term survives, the contribution of the WYD skew information, $I_{y}(\pi, D) \geq 0$, is to reduce the entropy production. This reduction is associated with the fluctuations in the operator $D$ and is one of the main results of this paper. It shows how quantum coherence, due to the noncommutativity of the thermodynamic charges, has a clear thermodynamic signature, effectively reducing the dissipation (entropy production) in the process. In particular, we see that the crucial ingredient for such a reduction is the quantum coherence between the observable $D$ associated with the currents and the global equilibrium state $\pi$. We introduce the relative reduction in entropy due to noncommutativity, which reads

$$
\mathcal{R}:=\frac{1}{2} \int_{0}^{1} d y \frac{I_{y}(\pi, D)}{\Sigma}=\frac{1}{2} \frac{\left\langle D^{2}\right\rangle_{\pi}}{\Sigma}-1,
$$

where we have used $\langle D\rangle_{\pi}=0$ and hence $\operatorname{var}(D)=\left\langle D^{2}\right\rangle_{\pi}$. The positivity of $I_{y}(\pi, D)$ and $\Sigma$ ensures $\mathcal{R} \geq 0$. This also leads to a universal upper bound to the entropy production, $\left\langle D^{2}\right\rangle_{\pi} / 2 \geq \Sigma$, which is saturated in the classical case, when $\mathcal{R}=0$.

The above results can be straightforwardly generalized to generic currents made by arbitrary combinations of the original charge currents in Eq. (6), $J_{k}^{\prime} \equiv \sum_{l} a_{k l} J_{l}$, with $a_{k l}$ arbitrary real coefficients. In such a case, the corresponding set of affinities $\lambda_{k}^{\prime}$ verify $\lambda_{l}=\sum_{k} a_{l k} \lambda_{l}^{\prime}$, leaving invariant the entropy production $\Sigma$ and the reduction $\mathcal{R}$ in Eq. (14). The Onsager matrix for the new currents then reads $\mathbb{L}^{\prime}=$ $\mathbb{A L} \mathbb{A}^{\dagger}$, where $\mathbb{A} \equiv\left\{a_{l k}\right\}$. As a consequence, we see that $\mathbb{L}^{\prime}$ continues to be symmetric and positive semidefinite.

\section{THERMOSQUEEZING OPERATIONS}

The general framework for non-Abelian transport introduced here allows us to explore a broad range of situations in quantum thermodynamics, where coherence and thermal effects are reciprocally coupled. To illustrate this point, we now consider the joint transport of energy and squeezing in interacting bosonic systems prepared in squeezed thermal states [19,20,40-43].

Let us focus first on the interaction between two resonant bosonic modes with frequency $\omega$, described by canonical variables $x_{i}, p_{i}$ satisfying $\left[x_{i}, p_{j}\right]=i \delta_{i j}$ (we set $\hbar=1$ ). Here $i=1,2$ refer to the two modes in question, which are prepared in a GGE of the form (1) with charges $Q_{1}^{(i)} \equiv H_{i}=\omega\left(p_{i}^{2}+x_{i}^{2}\right) / 2$, associated with energy, and $Q_{2}^{(i)} \equiv A_{i}=\omega\left(p_{i}^{2}-x_{i}^{2}\right) / 2$, associated with squeezing [20, 71]. These operators do not commute. In fact, together with $Q_{3}^{(i)}=\omega\left\{x_{i}, p_{i}\right\} / 2$, they form a $\mathrm{SU}(1,1)$ non-Abelian group. In analogy to the grand canonical ensemble, we identify the two affinities accompanying the charges as the inverse temperature $\lambda_{1} \equiv \beta$ (associated with the Hamiltonian $H$ ) and $\lambda_{2} \equiv-\beta \mu$ (associated with the squeezing asymmetry $A$ ), where $\mu$ is the equivalent of the chemical potential for squeezing currents (we do not include $Q_{3}^{(i)}$ among the charges of the GGE, merely for simplicity). The corresponding GGE is thus nothing but a squeezed thermal state [19], which can nowadays be prepared in many experimental platforms [72-74]. The amount of squeezing is customarily quantified by the squeezing parameter $r$, related to the affinities by $\mu=\tanh (2 r)=\lambda_{2} / \lambda_{1}$.

The interaction between the two modes must satisfy charge preservation, Eq. (3). We show in Appendix D that the only Gaussian unitary satisfying this property for two modes is a beamsplitter-type unitary of the form

$$
U=e^{-g \tau\left(a_{1}^{\dagger} a_{2}-a_{2}^{\dagger} a_{1}\right)},
$$

where $a_{i}=\left(x_{i}+i p_{i}\right) / \sqrt{2}$ are the ladder operators of the bosonic modes, $g$ is the interaction strength, and $\tau$ is the interaction time. To compute $\mathbb{L}$, we have developed a method based on the so-called symmetric logarithmic derivative (SLD). We note that the Onsager coefficients in Eq. (8) can be rewritten as

$$
L_{k i}=\frac{1}{2}\left\langle\left\{Q_{k}^{(1)}-\xi\left(Q_{k}^{(1)}\right), \Lambda_{i}\right\}\right\rangle_{\pi^{(1)}},
$$


where $\xi\left(Q_{k}^{(1)}\right) \equiv \operatorname{Tr}_{2}\left[\tilde{Q}_{k}^{(1)} \pi_{\lambda}^{(2)}\right]$ and we introduced the SLD $\Lambda_{i}$, defined by

$$
\left.\frac{1}{2}\left(\Lambda_{i} \pi_{\lambda}^{(1)}+\pi_{\lambda}^{(1)} \Lambda_{i}\right) \equiv \frac{\partial \pi_{\lambda+d \lambda}^{(1)}}{\partial\left(d \lambda_{i}\right)}\right|_{d \lambda_{i}=0} .
$$

The SLDs are Hermitian and can be defined for either subsystem. In the case of Abelian transport (commuting charges), they reduce to $\Lambda_{i}=\left\langle Q_{i}^{(1)}\right\rangle_{\pi^{(1)}}-Q_{i}^{(1)}$. For noncommuting charges, the SLD can be calculated from the following formula [75], valid for generic exponential quantum states:

$$
\Lambda_{i}=\left\langle Q_{i}^{(1)}\right\rangle_{\pi^{(1)}}-\sum_{n=0}^{\infty} f_{2 n} \mathcal{C}^{2 n}\left(Q_{i}^{(1)}\right)
$$

Here $\mathcal{C}^{m}(\mathcal{O}) \equiv[G, \ldots,[G, \mathcal{O}]]$ is the $m$ th-order nested commutator of an observable $\mathcal{O}$ with $G \equiv \sum_{\ell} \lambda_{\ell} Q_{\ell}$ [cf. Eq. (1)] and $\mathcal{C}^{0}(\mathcal{O})=\mathcal{O}$. In the above expression $f_{m}=4\left(4^{m / 2+1}-1\right) B_{m+2} /(m+2)$ !, where the $B_{k}$ are the Bernoulli numbers.

By identifying $Q_{1}^{(1)}=H^{(1)}$ and $Q_{2}^{(1)}=A^{(1)}$ in Eq. (16), we have $\lambda_{1}=\beta$ and $\lambda_{2}=-\beta \mu$ and the SLD can be obtained by calculating the nested commutators (see Appendix E). Using the SLD and the expressions for $Q_{k}^{(1)}-\xi\left(Q_{k}^{(1)}\right)$, we arrive at closed formulas for the Onsager coefficients:

$$
\begin{aligned}
L_{11}= & \sin ^{2}(g \tau) \frac{\omega^{2}}{1-\mu^{2}} \\
& \times\left[\bar{n}^{2}+\bar{n}+\mu^{2}\left(\frac{\tanh (\alpha)}{\alpha}\right)\left(\bar{n}^{2}+\frac{\bar{n}}{2}+\frac{1}{2}\right)\right],
\end{aligned}
$$

$$
\begin{aligned}
L_{12}= & \sin ^{2}(g \tau) \frac{\omega^{2} \mu}{1-\mu^{2}} \\
& \times\left[\bar{n}^{2}+\bar{n}+\left(\frac{\tanh (\alpha)}{\alpha}\right)\left(\bar{n}^{2}+\frac{\bar{n}}{2}+\frac{1}{2}\right)\right], \\
L_{22}= & \sin ^{2}(g \tau) \frac{\omega^{2}}{1-\mu^{2}} \\
& \times\left[\mu^{2}\left(\bar{n}^{2}+\bar{n}\right)+\left(\frac{\tanh (\alpha)}{\alpha}\right)\left(\bar{n}^{2}+\frac{\bar{n}}{2}+\frac{1}{2}\right)\right],
\end{aligned}
$$

and $L_{21}=L_{12}$. Here $\bar{n}=\left(e^{\alpha}-1\right)^{-1}$ and $\alpha=\beta \omega \sqrt{1-\mu^{2}}$. For no squeezing $(\mu=0)$, the cross-coefficients vanish, $L_{12}=L_{21}=0$. The above expressions can be simplified (but do not vanish) in both the high-temperature $(\beta \omega \ll 1)$ and the high-squeezing $(\mu \rightarrow 1)$ limits, where $\tanh (\alpha) / \alpha \rightarrow 1$. These results are plotted in Fig. 2. Notably, we find that coefficients are monotonically decreasing functions of $\beta$, for fixed $\mu$, and monotonically increasing functions of $\mu$, for fixed $\beta$. Note also that, in order to have nonzero transport, the unitary needs to be tuned to ensure that $g \tau \neq n \pi$ for $n=0,1,2, \ldots$, avoiding a complete swap.

In turn, we can use these results to compute $\Sigma$ and, in particular, the entropy reduction $\mathcal{R}$ in Eq. (14). The entropy production rate is given in terms of the Onsager coefficients in Eqs. (19) as

$$
\Sigma=L_{11} \delta \lambda_{1}^{2}+\left(L_{12}+L_{21}\right) \delta \lambda_{1} \delta \lambda_{2}+L_{22} \delta \lambda_{2}^{2},
$$

while the classical part of the entropy production is given from the variance of the operator $D$, as $\operatorname{var}(D)=\left\langle D^{2}\right\rangle_{\pi^{(1)}}$ (see Appendix E). Consequently, the relative entropy reduction $\mathcal{R}$ in Eq. (14) becomes

$$
\mathcal{R}=\frac{\alpha-\tanh (\alpha)}{2 \alpha /[3 \cosh (\alpha)-\sinh (\alpha)-1]+\tanh (\alpha)},
$$
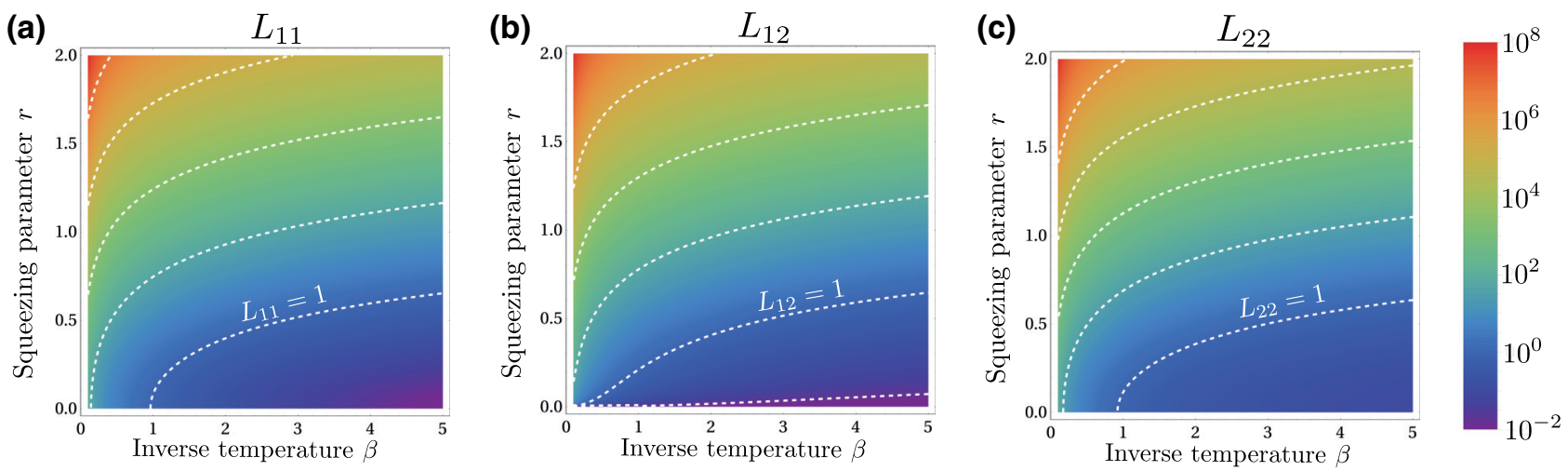

FIG. 2. (a)-(c) Thermosqueezing Onsager coefficients $L_{11}, L_{12}, L_{22}$ on the $\log$ scale, computed from Eqs. (19), in units of $(\hbar \omega)^{2} \sin ^{2}(g \tau)$, as a function of the inverse temperature $\beta$ (in units of $\hbar \omega / k_{B}$ ) and the adimensional squeezing parameter $r$. 

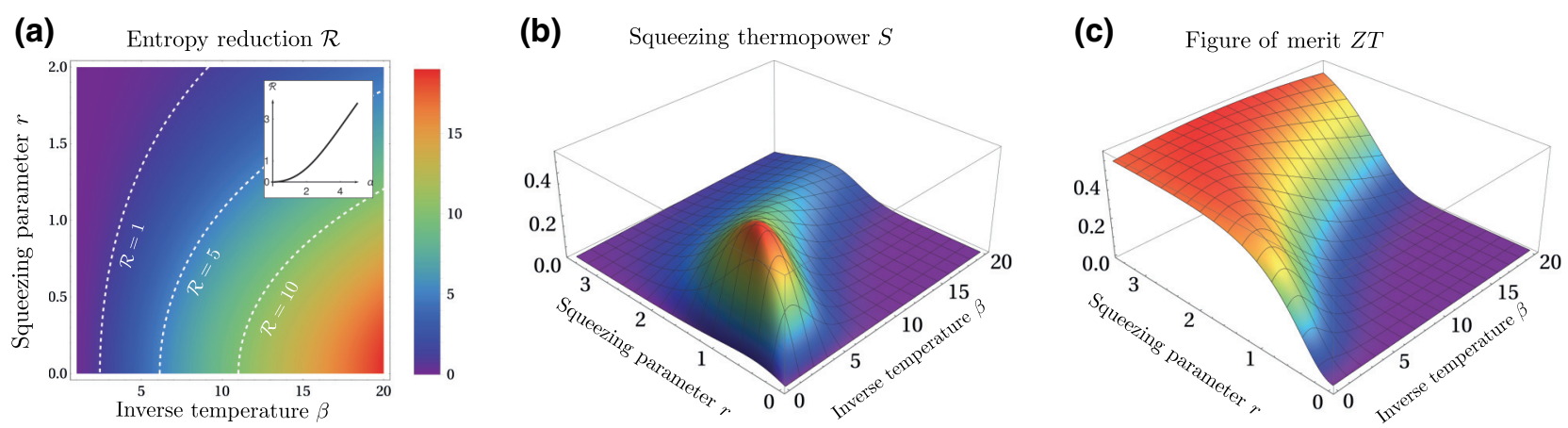

FIG. 3. (a) Entropy reduction $\mathcal{R}$, (b) squeezing thermopower coefficient $S$, and (c) figure of merit $Z T$ as a function of the inverse temperature $\beta$ and squeezing parameter $r$. The squeezing thermopower and $\beta$ are given in units of $\hbar \omega / k_{B}$ and $k_{B} / \hbar \omega$, respectively. Inset: entropy reduction as a function of the adimensional parameter $\alpha=\beta \omega \sqrt{1-\mu^{2}}$. In all plots we set $g \tau=\pi / 2$.

which in the high-temperature and high-squeezing limits, $\alpha \ll 1$, vanishes, i.e., $\mathcal{R} \rightarrow 0$. Remarkably, we find that $\mathcal{R}$ does not depend on the affinity gradients $\delta \lambda_{i}$, but it is only a function of the parameter $\alpha$ and hence depends only on the actual values of $\beta$ and $\mu$ (or $r$ ). In fact, even more than that, it depends on $\beta$ and $\mu$ only via the parameter $\alpha=\beta \omega \sqrt{1-\mu^{2}}$. It can therefore be considered as a generic property of the setup. In Fig. 3(a) we show $\mathcal{R}$ as a function of $\beta$ and $\mu$ (and as a function of $\alpha$ in the inset). Interestingly, while greater entropy reductions are obtained at low temperatures (where quantum effects become dominant), we observe that a high squeezing in the reservoir actually spoils the reduction effect.

\section{THERMOSQUEEZING EFFECTS}

In order to establish a connection with thermoelectric effects, we now focus on the Onsager coefficients for the transport of heat and squeezing. This can be done by noting that heat current is a linear combination of energy and squeezing currents [19], that is, $J_{Q}^{(i)} \equiv J_{1}^{(i)}-\mu_{i} J_{2}^{(i)}$, while $J_{A}^{(i)} \equiv J_{2}^{(i)}$. The corresponding affinities are the changes in inverse temperatures and squeezing potentials, $\delta \lambda_{1}^{\prime}=\delta \beta$ and $\delta \lambda_{2}^{\prime}=-\beta \delta \mu-\mu \delta \beta$. In this case we obtain the following reciprocity relations (see Appendix F for details):

$$
\begin{aligned}
& J_{Q}^{(1)}=L_{Q Q} \delta \beta-L_{Q A} \beta \delta \mu, \\
& J_{A}^{(1)}=L_{A Q} \delta \beta-L_{A A} \beta \delta \mu,
\end{aligned}
$$

with Onsager coefficients $\left(\mathbb{L}^{\prime} \geq 0\right)$

$$
\begin{aligned}
& L_{Q Q}=\omega^{2} \sin ^{2}(g \tau)\left(1-\mu^{2}\right) \bar{n}(\bar{n}+1), \\
& L_{Q A}=\omega^{2} \sin ^{2}(g \tau) \mu \bar{n}(\bar{n}+1),
\end{aligned}
$$

$$
\begin{aligned}
L_{A A}= & \omega^{2} \sin ^{2}(g \tau)\left(\frac{1}{1-\mu^{2}}\right) \\
& \times\left[\mu^{2} \bar{n}(\bar{n}+1)+\left(\frac{\tanh (\alpha)}{\alpha}\right)\left(\bar{n}^{2}+\frac{\bar{n}}{2}+\frac{1}{2}\right)\right],
\end{aligned}
$$

and $L_{A Q}=L_{Q A}$. In the absence of squeezing $(\mu \rightarrow 0)$, the cross-coefficients vanish, $L_{Q A}, L_{A Q} \rightarrow 0$. The behavior of these quantities with $\beta$ and $\mu$ is similar to those of Eqs. (19): monotonically decreasing with $\beta$ and increasing with $\mu$.

Coefficients (23) can be related to cross-effects linking transport in heat and squeezing, in analogy to thermoelectric coefficients [1]. We start by characterizing the coefficients $L_{Q Q}$ and $L_{A A}$ by defining thermal and squeezing conductance. These relate heat and squeezing asymmetry currents to the corresponding gradients in temperature $\delta T=T_{1}-T_{2}$ (at constant squeezing) and squeezing potentials $\delta \mu=\mu_{1}-\mu_{2}$ (at constant temperature) as

$$
\begin{gathered}
\kappa \equiv\left(\frac{J_{Q}}{\delta T}\right)_{\delta \mu=0}=-\frac{L_{Q Q}}{T^{2}}, \\
G \equiv\left(\frac{J_{A}}{\delta \mu}\right)_{\delta T=0}=-\frac{L_{A A}}{T},
\end{gathered}
$$

where $\kappa$ is the thermal conductance and $G$ is analogous to the electric conductance for squeezing flows. We have also used the fact that $\delta \beta=-\delta T / T^{2}$. Using Eqs. (24) and (25), the total heat dissipated in the setup [76] reads

$$
\dot{Q} \equiv T \Sigma=\kappa \delta T^{2} / T+J_{A}^{2} / G
$$

The first term represents the heat dissipated by the thermal resistance, while the second is a Joule-like heating due to the squeezing current.

The squeezing thermopower (or squeezing-Seebeck coefficient) measures the squeezing potential difference 
$\delta \mu$ developed between reservoirs due to a difference in temperatures $\delta T$ when no net squeezing current is flowing:

$$
S \equiv-\left(\frac{\delta \mu}{\delta T}\right)_{J_{A}=0}=\frac{1}{T} \frac{L_{A Q}}{L_{A A}} .
$$

Analogously, the squeezing-Peltier coefficient measures the heat current $J_{Q}$ produced by a squeezing current $J_{A}$ in isothermal conditions $(\delta T=0)$ :

$$
\Pi \equiv\left(\frac{J_{Q}}{J_{A}}\right)_{\delta T=0}=\frac{L_{Q A}}{L_{A A}} .
$$

The symmetry of the coefficients leads to a relation between squeezing Seebeck and Peltier effects $\Pi=T S$.

A precise determination of the thermosqueezing coefficients, Eqs. (24)-(28), may be useful to a number of applications. For example, assuming that the squeezing thermopower $S$ is known, Eq. (27) allows the development of a squeezing-based thermometer. Closely following the principle on which thermocouples are based, temperature differences can be obtained from squeezing potential differences (or the other way around) through the relation $\delta T=-(1 / S) \delta \mu$, valid when $J_{A} \rightarrow 0$. This requires tuning the device to obtain zero squeezing current between the reservoirs. Possibilities to implement such "opencircuit" conditions may include introducing counterterms in the interaction $U$ to effectively cancel the exchange of squeezing (e.g., applying local squeezing unitaries on each mode after the collision), or to interpose a small interface between the reservoirs blocking the squeezing current, while allowing the exchange of heat [77]. In Fig. 3(b) we show the dependence of $S$ with respect to the temperature $\beta$ and squeezing parameter $r$. The small values of $S$ obtained suggest that tiny differences in squeezing potentials may be detected by measuring the larger temperature gradient.

The analogy with thermoelectricity also allow us to analyze this setup as a thermosqueezing engine. The squeezing current $J_{A}>0$ can be used to power a heat flux $J_{Q}<0$ against the temperature bias $\delta \beta>0$, hence refrigerating the cold reservoir, when the difference in squeezing potentials is sufficiently high, $\delta \mu \geq \delta \mu_{\mathrm{fr}} \equiv \kappa T \delta \beta /(G S)$. The work (per collision) consumed by such a fridge is given by $\dot{W}=J_{A} \delta \mu$, and can be made negative (work extraction) in the regime $0 \leq \delta \mu \leq \delta \mu_{\text {stop }} \equiv \Pi(\delta \beta / \beta)$, where $J_{Q}>0$. In the linear response regime, maximum power is obtained at $\delta \mu_{\text {stop }} / 2$, while maximum efficiency in both the refrigerator and engine regimes, as well as efficiency at maximum power, are determined by the adimensional figure of merit

$$
Z T \equiv \frac{G S^{2} T}{\kappa},
$$

defined in analogy to standard thermoelectrics $[47,76,78]$. In Fig. 3(c) we show $Z T$ as a function of the squeezing parameter for different values of the temperature. It quickly saturates when increasing the squeezing parameter at any temperature, leading to values $Z T \simeq 0.5$, corresponding to $\eta\left(\dot{W}_{\max }\right) \simeq \eta_{C} / 10$, where $\eta_{C} \equiv 1-\beta_{1} / \beta_{2}$ is the Carnot efficiency. This result can be intuitively understood from the fact that, since $[H, A] \neq 0$, heat and asymmetry currents can never be proportional to each other, hence avoiding the so-called tight coupling condition $[47,76,78]$.

\section{CONCLUSIONS}

We formulated a general framework based on a collisional model, and derived Onsager reciprocity relations for non-Abelian transport in linear response. Our results lead to the identification of an entropy reduction effect induced by quantum coherence and the noncommutativity of thermodynamic charges. We illustrated our findings by developing reciprocal relations between energy and squeezing in bosonic setups. The applications of such reciprocal theory may be of use to a variety of quantum information technologies and enable new ways to convert heat into work in the quantum realm.

Our results are within reach of current experimental devices employed in thermodynamic scenarios, like the trapped ion platform used in Ref. [79], where different motional modes can be weakly coupled through Raman beams and prepared in squeezed thermal states reaching values up to $r \sim 1.5$. Another option would be building upon the squeezing-based nanobeam engine in Ref. [41], able to reach squeezings around $r \sim 0.5$ at room temperatures. The quantum limit can be instead achieved in other setups for micromechanical oscillators by using reservoirengineering techniques like two-tone driving [72,73]. A third possibility would be to consider setups combining optical cavities and ultracold atoms [80,81]. In Ref. [80] ponderomotive squeezing has been achieved in optical modes using an atomic cloud, while in Ref. [81] a Bose gas interacts simultaneously with two optical cavities. Coupling the device to an extra cavity in the former case, and by appropriately pumping the cavities with squeezed radiation in the latter, one could effectively implement a thermosqueezing device and thus also explore some of the consequences presented in this framework.

Extensions to situations with multiple terminals and including external modulation may be important in view of applications, in close analogy with thermoelectricity [8284]. Of particular interest would be to consider squeezing in the context of hybrid thermal machines operating with multiple conserved quantities [85], as well as the study of fluctuations in the currents for non-Abelian setups, where standard exchange fluctuation theorems break down [86,87]. Finally, our theory should also lead to fruitful applications in other non-Abelian quantum systems $[15,24]$. 


\section{ACKNOWLEDGMENTS}

We thank J.P. Santos for fruitful discussions. The authors acknowledge support from the Abdus Salam International Centre of Theoretical Physics, where part of this work was developed, for both hospitality and financial support. G.M. acknowledges funding from Spanish MICINN through the Juan de la Cierva program (IJC2019-039592I), the European Union's Horizon 2020 research and innovation program under the Marie Skłodowska-Curie Grant Agreement No. 801110, and the Austrian Federal Ministry of Education, Science and Research (BMBWF). J.M.R.P. acknowledges financial support from the Spanish Government (Grant Contract FIS-2017-83706-R) and from the Foundational Questions Institute Fund, a donor-advised fund of Silicon Valley Community Foundation (Grant No. FQXi-IAF19-01). G.T.L. acknowledges the hospitality of Apt. 44, where part of this work was developed, and the financial support of the São Paulo Funding Agency FAPESP (Grants No. 2017/50304-7, No. 2017/07973-5, and No. 2018/12813-0) and the Brazilian funding agency CNPq (Grant No. INCT-IQ 246569/2014-0).

\section{APPENDIX A: INFORMATIONAL FORM OF THE ENTROPY PRODUCTION RATE}

Equation (5) in Sec. II expresses the entropy production rate per collision in terms of the charge fluxes and thermodynamic forces. Here we show that such an expression is equivalent to the fully informational form for entropy production derived in Refs. [33,66-68] and show its positivity. Following the informational approach, the total entropy production due to a single collision can be written as

$$
\begin{aligned}
\Sigma & =\Delta S_{1}+\Delta S_{2}+S\left(\rho_{1}^{\prime} \| \pi_{\lambda^{(1)}}^{(1)}\right)+S\left(\rho_{2}^{\prime} \| \pi_{\lambda^{(2)}}^{(2)}\right) \\
& =\mathcal{I}\left(\rho_{12}^{\prime}\right)+S\left(\rho_{1}^{\prime} \| \pi_{\lambda^{(1)}}^{(1)}\right)+S\left(\rho_{2}^{\prime} \| \pi_{\lambda^{(2)}}^{(2)}\right) \\
& \geq 0
\end{aligned}
$$

where $\Delta S_{i}=S\left(\rho_{i}^{\prime}\right)-S\left(\pi_{\lambda^{(i)}}^{(i)}\right)$ represents the change in (local) von Neumann entropy of subsystem $i=1,2$ due to the interaction, and $S(\rho \| \sigma)=\operatorname{Tr}(\rho \ln \rho-\rho \ln \sigma)$ is the quantum relative entropy, accounting for the entropic cost necessary for resetting the subsystems back to equilibrium, i.e., for maintaining the reservoirs in equilibrium [66]. In the second equality we introduced the mutual information $\mathcal{I}\left(\rho_{12}^{\prime}\right)=S\left(\rho_{1}^{\prime}\right)+S\left(\rho_{2}^{\prime}\right)-S\left(\rho_{12}^{\prime}\right)$ developed between systems 1 and 2 due to map (2). Since the initial state is a product state and the interaction is unitary, it follows that $\mathcal{I}\left(\rho_{12}^{\prime}\right)=\Delta S_{1}+\Delta S_{2}$. By expressing the entropy production in the form (A1) the second law is automatically satisfied, $\Sigma \geq 0$, since each term in Eq. (A1) is individually non-negative.
Using the explicit expressions for the entropy changes $\Delta S_{i}=S\left(\rho_{i}^{\prime}\right)-S\left(\pi_{\lambda^{(i)}}^{(i)}\right)$ and the relative entropy contributions $S\left(\rho_{i}^{\prime} \| \pi_{\lambda^{(i)}}^{(i)}\right)=\operatorname{Tr}\left(\rho_{i}^{\prime} \ln \rho_{i}^{\prime}-\rho \ln \pi_{\lambda^{(i)}}^{(i)}\right)$, we arrive at the form

$$
\Sigma=\operatorname{Tr}\left[\left(\pi_{\lambda^{(1)}}^{(1)}-\rho_{1}^{\prime}\right) \ln _{\lambda^{(1)}}^{(1)}\right]+\operatorname{Tr}\left[\left(\pi_{\lambda^{(2)}}^{(2)}-\rho_{2}^{\prime}\right) \ln _{\lambda^{(2)}}^{(2)}\right]
$$

which, upon substituting for the GGE in Eq. (1), leads exactly to Eq. (5). Finally, we stress that, since every collision starts with the same product of initial GGE states $\pi_{\lambda^{(1)}}^{(1)} \otimes \pi_{\lambda^{(2)}}^{(2)}$ and is subjected to the same unitary interaction $U$, the expression in Eq. (A2) represents the entropy production rate (per collision).

\section{APPENDIX B: PROOF OF THEOREM 1}

In this appendix we provide a detailed proof of Theorem 1. Recall that we are assuming that $\lambda_{k}^{(2)}=\lambda_{k}$ but $\lambda_{k}^{(1)}=$ $\lambda_{k}+\delta \lambda_{k}$, where the $\delta \lambda_{k}$ are small real numbers. We use the following Feynman integral representations, valid for an arbitrary operator $G(\phi)$, depending on some parameter $\phi$ :

$$
\partial_{\phi} e^{-G}=-\int_{0}^{1} d y e^{-G y}\left(\partial_{\phi} G\right) e^{-G(1-y)} .
$$

Applying this to $\pi^{(1)}$, as given in Eq. (1), we find that

$$
\begin{aligned}
\left.\frac{\partial \pi_{\lambda+\delta \lambda}^{(1)}}{\partial\left(\delta \lambda_{\ell}\right)}\right|_{\delta \lambda_{\ell}=0}= & \left\langle Q_{\ell}^{(1)}\right\rangle_{\pi^{(1)}} \pi^{(1)} \\
& -\int_{0}^{1} d y\left(\pi_{\lambda}^{(1)}\right)^{y} Q_{\ell}^{(1)}\left(\pi_{\lambda}^{(1)}\right)^{1-y},
\end{aligned}
$$

where we have also used the fact that

$$
-\left.\frac{\partial \ln Z_{1}}{\partial\left(\delta \lambda_{\ell}\right)}\right|_{\delta \lambda_{\ell}=0}=\left\langle Q_{\ell}^{(1)}\right\rangle_{\pi}
$$

The above expressions can be rewritten by taking the tensor product with $\pi_{\lambda}^{(2)}$. We then get

$$
\begin{aligned}
\left.\frac{\partial \pi_{\lambda+\delta \lambda}^{(1)}}{\partial\left(\delta \lambda_{\ell}\right)}\right|_{\delta \lambda_{\ell}=0} \otimes \pi_{2} & =\left\langle Q_{\ell}^{(1)}\right\rangle_{\pi} \pi-\int_{0}^{1} d y \pi^{y} Q_{\ell}^{(1)} \pi^{1-y} \\
& =\left\langle Q_{\ell}^{(1)}\right\rangle_{\pi} \pi-\int_{0}^{1} d y \pi^{1-y} Q_{\ell}^{(1)} \pi^{y},
\end{aligned}
$$

where $\pi=\pi_{\lambda}^{(1)} \otimes \pi_{\lambda}^{(2)}$ and, in the second equality, we simply changed variables from $y$ to $1-y$. We write these two forms here, side by side, as they will be useful in understanding the conditions for $L_{k \ell}$ to be symmetric. 
Inserting Eq. (B2) into Eq. (10) leads to the two forms for the Onsager matrix,

$$
\begin{aligned}
L_{k \ell} & =-\int_{0}^{1} d y \operatorname{Tr}\left[\left(\tilde{Q}_{k}^{(1)}-Q_{k}^{(1)}\right) \pi^{y} Q_{\ell}^{(1)} \pi^{1-y}\right] \\
& =-\int_{0}^{1} d y \operatorname{Tr}\left[\left(\tilde{Q}_{k}^{(1)}-Q_{k}^{(1)}\right) \pi^{1-y} Q_{\ell}^{(1)} \pi^{y}\right]
\end{aligned}
$$

where we have used the fact that terms of the form $\left\langle Q_{\ell}^{(1)}\right\rangle_{\pi}\left\langle\tilde{Q}_{k}^{(1)}-Q_{k}^{(1)}\right\rangle_{\pi}$ vanish since $U \pi U^{\dagger}=\pi$.

Equations (B3a) and (B3b) provide two equivalent ways of writing the Onsager coefficients. We now prove that, when the charges, and the dynamics, are time-reversal invariant, the matrix $L$ is symmetric, i.e., $L_{\ell k}=L_{k \ell}$. To accomplish this, we essentially exchange $k \leftrightarrow \ell$ in the first equality in Eq. (B3) and compare with the second one. Indeed, exchanging $k \leftrightarrow \ell$ and using the cyclic property of the trace, we obtain

$$
\begin{aligned}
L_{\ell k}= & -\int_{0}^{1} d x \operatorname{Tr}\left[Q_{k}^{(1)} \pi^{1-x} \tilde{Q}_{\ell}^{(1)} \pi^{x}\right] \\
& +\int_{0}^{1} d x \operatorname{Tr}\left[Q_{k}^{(1)} \pi^{1-x} Q_{\ell}^{(1)} \pi^{x}\right] .
\end{aligned}
$$

We note that the second term is already the same as the last term of the second equality in Eq. (B3). We thus need to focus only on the first term. Since $\pi$ is a fixed point of $U$, we can write the first term above as

$$
\operatorname{Tr}\left[Q_{k}^{(1)} \pi^{1-x} \tilde{Q}_{\ell}^{(1)} \pi^{x}\right]=\operatorname{Tr}\left[U Q_{k}^{(1)} U^{\dagger} \pi^{1-x} Q_{\ell}^{(1)} \pi^{x}\right],
$$

where we stress that $U Q_{k}^{(1)} U^{\dagger} \neq \tilde{Q}_{k}^{(1)}$. We now introduce the time-reversal operator in quantum mechanics $\Theta$, responsible for changing the sign of odd variables under time inversion, such as momenta and the magnetic field $[88,89]$. Using it, we can define the time reversal of the unitary evolution $\bar{U}=\Theta U^{\dagger} \Theta^{\dagger}$ and time reversal of the charges as $\bar{Q}_{k}^{(1)}=\Theta Q_{k}^{(1)} \Theta^{\dagger}$. Introducing these operators in Eq. (B5), it then follows that

$$
\begin{aligned}
\operatorname{Tr} & {\left[U Q_{k}^{(1)} U^{\dagger} \pi^{1-x} Q_{\ell}^{(1)} \pi^{x}\right] } \\
& =\operatorname{Tr}\left[\bar{U}^{\dagger} \bar{Q}_{k}^{(1)} \bar{U} \Theta \pi^{1-x} \Theta^{\dagger} \bar{Q}_{\ell}^{(1)} \Theta \pi^{x} \Theta^{\dagger}\right] .
\end{aligned}
$$

If the dynamics is time-reversal invariant then $\bar{U}=U$ is verified. Moreover, if the charges are time-reversal invariant then $\bar{Q}_{k}^{(1)}=Q_{k}^{(1)}$ and $\Theta \pi \Theta=\pi$. Using this in Eq. (B6), we finally arrive at

$$
\operatorname{Tr}\left[Q_{k}^{(1)} \pi^{1-x} \tilde{Q}_{\ell}^{(1)} \pi^{x}\right]=\operatorname{Tr}\left[\tilde{Q}_{k}^{(1)} \pi^{1-x} Q_{\ell}^{(1)} \pi^{x}\right] .
$$

Substituting Eq. (B7) into Eq. (B4) and comparing with (B3b) then clearly shows that $L_{\ell k}=L_{k \ell}$.
We finally express the results in terms of the $y$ covariance, defined in Eq. (9) of Sec. III. Comparing Eq. (9) with Eq. (B3a) immediately shows us that we can write

$$
L_{k \ell}=\int_{0}^{1} d y \operatorname{cov}_{y}\left(Q_{k}^{(1)}-\tilde{Q}_{k}^{(1)}, Q_{\ell}^{(1)}\right)
$$

Now using the fact that $L$ is symmetric, we can also write the above equation as an average between $L_{k \ell}$ and $L_{\ell k}$, leading to

$$
\begin{aligned}
L_{k \ell} & =\frac{1}{2}\left(L_{k \ell}+L_{\ell k}\right) \\
& =\frac{1}{2} \int_{0}^{1} d y \operatorname{cov}_{y}\left(\tilde{Q}_{k}^{(1)}-Q_{k}^{(1)}, \tilde{Q}_{\ell}^{(1)}-Q_{\ell}^{(1)}\right),
\end{aligned}
$$

which is Eq. (8) in Theorem 1.

\section{APPENDIX C: ONSAGER-CASIMIR RECIPROCITY}

As stressed in Sec. III, Onsager reciprocity follows from stationarity of the dynamics, $U \pi U^{\dagger}=\pi$, and invariance under time reversal, $\bar{U}=U$ and $\bar{Q}=Q$. However, the second assumption can be relaxed to obtain a weaker form of reciprocity, named Onsager-Casimir reciprocity [69].

In this case time inversion of the dynamics leads to a different evolution, $\bar{U}=U_{*}$, and potentially different charges $\bar{Q}_{k}^{(1)}=Q_{k}^{*(1)}$, depending on the parity of the operators $Q_{k}^{(1)}$. In the evolution $U_{*}$ and charges $Q_{k}^{*(1)}$ external magnetic fields and/or other odd variables change their signs. Using these relations in Eq. (B6), it follows that

$$
\operatorname{Tr}\left[Q_{k}^{(1)} \pi^{1-x} \tilde{Q}_{\ell}^{(1)} \pi^{x}\right]=\operatorname{Tr}\left[\tilde{Q}_{k}^{*(1)} \pi_{*}^{1-x} Q_{\ell}^{*(1)} \pi_{*}^{x}\right],
$$

where $\tilde{Q}_{k}^{*(1)}=U_{*}^{\dagger} Q_{\ell}^{*(1)} U_{*}$ and $\pi_{*}=e^{-\sum_{k} \lambda_{k} Q_{k}^{*}} / Z_{*}=\pi_{\lambda}^{*(1)}$ $\otimes \pi_{\lambda}^{*(2)}$ is the fixed point of the evolution, $U_{*} \pi_{*} U_{*}^{\dagger}=\pi_{*}$. Introducing the time-reversal operator in the second term of Eq. (B4) we obtain

$$
L_{\ell k}=L_{k \ell}^{*},
$$

where the transport coefficients $L_{k \ell}^{*}$ are defined similarly to $L_{k \ell}$, but with respect to the modified evolution $U_{*}$ and charges $Q_{k}^{*(1)}$ in the GGE $\pi_{\lambda+\delta \lambda}^{*(1)}$, that is,

$$
L_{k \ell}^{*}=\operatorname{Tr}\left[\left(\tilde{Q}_{k}^{*(1)}-Q_{k}^{*(1)}\right)\left(\left.\frac{\partial \pi_{\lambda+\delta \lambda}^{*(1)}}{\partial\left(\delta \lambda_{\ell}\right)}\right|_{\delta \lambda_{\ell}=0} \otimes \pi_{\lambda}^{*(2)}\right)\right] .
$$

\section{APPENDIX D: GENERAL GAUSSIAN THERMOSQUEEZING OPERATIONS}

In this appendix we discuss the general structure of thermosqueezing operations that preserve Gaussianity. We 
assume that systems 1 and 2 are composed of arbitrary numbers $N_{1}$ and $N_{2}$ of bosonic modes. Let $\mathcal{N}_{\alpha}$ denote the set of modes belonging to system $\alpha=1,2$. We then define the net charges $Q_{1,2,3}$ associated with each system as

$$
\begin{gathered}
Q_{1}^{(\alpha)}=\frac{\hbar \omega}{2} \sum_{i \in \mathcal{N}_{\alpha}}\left(p_{i}^{2}+x_{i}^{2}\right) \quad Q_{2}^{(\alpha)}=\frac{\hbar \omega}{2} \sum_{i \in \mathcal{N}_{\alpha}}\left(p_{i}^{2}-x_{i}^{2}\right) \\
Q_{3}^{(\alpha)}=\frac{\hbar \omega}{2} \sum_{i \in \mathcal{N}_{\alpha}}\left\{x_{i}, p_{i}\right\} .
\end{gathered}
$$

We are interested here in Gaussian operations satisfying the charge preservation condition (3). Let $\mathbf{R}=$ $\left(x_{1}, x_{2}, \ldots, p_{1}, p_{2}, \ldots\right)$ denote a vector of size $2 N$ (where $N=N_{1}+N_{2}$ ) containing all position operators of systems 1 and 2, followed by all momentum operators of systems 1 and 2. A generic Gaussian unitary can then be translated into the symplectic transformation

$$
U^{\dagger} R_{i} U=\sum_{j} V_{i j} R_{j},
$$

where $V$ is a $2 N$-dimensional symplectic matrix, satisfying

$$
V^{T} \Omega V=\Omega
$$

with

$$
\Omega=\left(\begin{array}{cc}
0 & \mathbb{1}_{N} \\
-\mathbb{1}_{N} & 0
\end{array}\right)
$$

being the symplectic form and $\mathbb{1}_{N}$ the identity of dimension $N$.

Our goal now is to obtain the additional restrictions (besides being symplectic) that are imposed on $V$ if it is to satisfy the charge preservation condition (3). We begin by rewriting Eq. (3) as

$$
U^{\dagger}\left(Q_{i}^{(1)}+Q_{i}^{(2)}\right) U=Q_{i}^{(1)}+Q_{i}^{(2)} .
$$

Next we note that, since the $Q_{i}^{(\alpha)}$ are quadratic in the vector $\mathbf{R}$, we can rewrite them as

$$
Q_{i}^{(1)}+Q_{i}^{(2)}=\frac{\hbar \omega}{2} \sum_{m, n}\left(K_{i}\right)_{m n} R_{m} R_{n}
$$

where $K_{i}$ for $i=1,2,3$ are $2 N$-dimensional matrices reading

$$
K_{1}=\mathbb{1}_{2 N}, \quad K_{2}=\left(\begin{array}{cc}
\mathbb{1}_{N} & 0 \\
0 & -\mathbb{1}_{N}
\end{array}\right), \quad K_{3}=\left(\begin{array}{cc}
0 & \mathbb{1}_{N} \\
\mathbb{1}_{N} & 0
\end{array}\right) .
$$

Using Eq. (D1), we may now recast Eq. (D3) in the form

$$
V^{T} K_{i} V=K_{i}
$$

This can be viewed as the symplectic counterpart of the Hilbert space identity (D3). Together with Eq. (D2), it yields a total of four restrictions on the form of $V$.
Energy preservation is related to $K_{1}$. As a consequence, in addition to being symplectic, Eq. (D5) determines that $V$ must also be orthogonal, that is, $V^{T} V=\mathbb{1}_{2 N}$. As shown, e.g., in Ref. [90], the intersection of the symplectic and orthogonal groups are matrices of the form

$$
O(2 N) \cap \operatorname{Sp}(2 N)=\left\{\left(\begin{array}{cc}
X & Y \\
-Y & X
\end{array}\right) \mid X-i Y=U(N)\right\},
$$

where $U(N)$ is the group of unitary matrices. All symplectic forms preserving $Q_{1}$ must therefore be of this form.

On top of this, we now impose the conditions in Eq. (D5) for preservation of squeezing, $Q_{2}$ and/or $Q_{3}$. As one can verify, these will only be satisfied if $Y=0$, independently of whether we impose the conservation of only $Q_{2}, Q_{3}$, or both of them. We thus finally conclude that the most general thermosqueezing operation has the form

$$
V=\left(\begin{array}{rr}
X & 0 \\
0 & X
\end{array}\right), \quad X^{T} X=1
$$

The matrix $X$ must be real and also a member of $U(N)$, which is tantamount to $X \in O(N)$, the group of orthogonal matrices of size $N$. We remark that Eq. (D7) is written with respect to the ordering $\mathbf{R}=\left(x_{1}, x_{2}, \ldots, p_{1}, p_{2}, \ldots\right)$. If one wishes to use the more standard ordering $\tilde{\mathbf{R}}=$ $\left(x_{1}, p_{1}, x_{2}, p_{2}, \ldots\right)$ then the entries of $V$ must be rearranged appropriately.

The particular case employed in our example, Eq. (15), corresponds to $N_{1}=N_{2}=1$; that is, each system is composed of only a single mode. In this case the matrix $X$ will be of size $N=N_{1}+N_{2}=2$ and hence can be parametrized as

$$
X=\left(\begin{array}{cc}
\cos \phi & -\sin \phi \\
\sin \phi & \cos \phi
\end{array}\right)
$$

which is quantified by a single parameter $\phi$. This corresponds precisely to the unitary

$$
U=e^{-i g \tau\left(x_{1} p_{2}-x_{2} p_{1}\right)}=e^{-g \tau\left(a_{1}^{\dagger} a_{2}-a_{2}^{\dagger} a_{1}\right)}
$$

with the identification $\phi=g \tau$, where $g$ is the interaction strength and $\tau$ is the interaction time. Interestingly, this analysis also shows that unitary (D9) is the only two-mode Gaussian unitary preserving both energy and squeezing.

We also call attention to the phase appearing in Eq. (D9). An interaction of the form $e^{-i g\left(a_{1}^{\dagger} a_{2}+a_{2}^{\dagger} a_{1}\right)}$, for instance, conserves energy, but does not conserve squeezing. What the results above show is that $i\left(a_{1}^{\dagger} a_{2}-a_{2}^{\dagger} a_{1}\right)$ is the only type of interaction conserving both energy and squeezing. 


\section{APPENDIX E: ONSAGER COEFFICIENTS FOR ENERGY AND SQUEEZING}

In this appendix we give details on the calculation of the Onsager matrix $\mathbb{L}$ using the SLD, as well as the contributions to the entropy production. As mentioned in Sec. IV, identifying $Q_{1}^{(1)}=H^{(1)}$ and $Q_{2}^{(1)}=A^{(1)}$ in Eq. (16) yields $\lambda_{1}=\beta$ and $\lambda_{2}=-\beta \mu$. Using Eq. (18) and calculating the nested commutators, we arrive at

$$
\begin{aligned}
& \Lambda_{1}=\langle H\rangle_{\pi^{(1)}}-\left(H+\left[\frac{\tanh (\alpha)}{\alpha}-1\right] \frac{\mu}{1-\mu^{2}}(A-\mu H)\right), \\
& \Lambda_{2}=\langle A\rangle_{\pi^{(1)}}-\left(A+\left[\frac{\tanh (\alpha)}{\alpha}-1\right] \frac{1}{1-\mu^{2}}(A-\mu H)\right),
\end{aligned}
$$

where we have used $\sum_{n=1}^{\infty} f_{2 n} \alpha^{2 n}=\tanh (\alpha) / \alpha$ and we recall that $\alpha=\beta \hbar \omega \sqrt{1-\mu^{2}}$. In the high-temperature $(\beta \omega \ll 1)$ or high-squeezing $(\mu \rightarrow 1)$ limits, we have $\alpha \ll 1$ and hence $\tanh (\alpha) / \alpha \rightarrow 1$. In such a case we recover the expressions for the SLDs in the Abelian case.

For the quantities $Q_{k}^{(1)}-\tilde{Q}_{k}^{(1)}$, we obtain

$$
\begin{aligned}
Q_{1}^{(1)}-\tilde{Q}_{1}^{(1)}= & \sin ^{2}(g \tau)\left(H^{(1)}-H^{(2)}\right) \\
& -\cos (g \tau) \sin (g \tau) \omega\left(a_{1}^{\dagger} a_{2}+a_{1} a_{2}^{\dagger}\right), \\
Q_{2}^{(1)}-\tilde{Q}_{2}^{(1)}= & \sin ^{2}(g \tau)\left(A^{(1)}-A^{(2)}\right) \\
& -\cos (g \tau) \sin (g \tau) \omega\left(a_{1} a_{2}+a_{1}^{\dagger} a_{2}^{\dagger}\right) .
\end{aligned}
$$

Here $a_{i}$ for $i=1,2$ are the ladder operators of the two bosonic modes. Taking the trace over mode 2 in the equilibrium state $\pi_{\lambda}^{(2)}$ leads to

$$
\begin{aligned}
& Q_{1}^{(1)}-\xi\left(Q_{1}^{(1)}\right)=\sin ^{2}(g \tau)\left(H^{(1)}-\left\langle H^{(1)}\right\rangle_{\pi^{(1)}}\right), \\
& Q_{2}^{(1)}-\xi\left(Q_{2}^{(1)}\right)=\sin ^{2}(g \tau)\left(A^{(1)}-\left\langle A^{(1)}\right\rangle_{\pi^{(1)}}\right),
\end{aligned}
$$

where we took advantage of the fact that in equilibrium $\left\langle Q_{k}^{(1)}\right\rangle_{\pi^{(1)}}=\left\langle Q_{k}^{(2)}\right\rangle_{\pi^{(2)}}$.

Introducing the SLDs in Eqs. (E1) and expressions (E3) into Eq. (16) we arrive at the Onsager coefficients in Eqs. (19). In order to arrive at that final form, we also used the relations

$$
\begin{aligned}
\left\langle H^{2}\right\rangle_{\pi} & =\cosh ^{2}(2 r)\left\langle H^{2}\right\rangle_{\mathrm{th}}+\sinh ^{2}(2 r)\left\langle A^{2}\right\rangle_{\mathrm{th}}, \\
\left\langle A^{2}\right\rangle_{\pi} & =\cosh ^{2}(2 r)\left\langle A^{2}\right\rangle_{\mathrm{th}}+\sinh ^{2}(2 r)\left\langle H^{2}\right\rangle_{\mathrm{th}}, \\
\langle H A\rangle_{\pi} & =\langle A H\rangle_{\pi} \\
& =\sinh (2 r) \cosh (2 r)\left(\left\langle H^{2}\right\rangle_{\mathrm{th}}+\left\langle A^{2}\right\rangle_{\mathrm{th}}\right), \\
\langle H\rangle_{\pi}^{2} & =\cosh ^{2}(2 r)\left\langle H^{2}\right\rangle_{\mathrm{th}}^{2}, \\
\langle A\rangle_{\pi}^{2} & =\sinh ^{2}(2 r)\left\langle H^{2}\right\rangle_{\mathrm{th}}^{2}, \\
\langle H\rangle_{\pi}\langle A\rangle_{\pi} & =\sinh (2 r) \cosh (2 r)\left(\langle H\rangle_{\mathrm{th}}^{2}+\langle A\rangle_{\mathrm{th}}^{2}\right),
\end{aligned}
$$

where $\left\langle Q_{i}\right\rangle_{\text {th }}=\operatorname{Tr}\left[Q_{i} e^{-\alpha H / \omega} / Z_{\alpha}\right]$ are thermal averages in a Gibbs state at temperature $\alpha / \omega$, and we omitted the superscripts $(i)$ in $H, A$, and $\pi$ since the expressions are the same for modes 1 or 2 . The explicit expressions for the thermal averages introduced above are

$$
\begin{aligned}
\left\langle H^{2}\right\rangle_{\text {th }} & =(\hbar \omega)^{2}\left(2 \bar{n}^{2}+2 \bar{n}+1 / 4\right), \\
\left\langle A^{2}\right\rangle_{\text {th }} & =(\hbar \omega)^{2}\left(\bar{n}^{2}+\bar{n} / 2+1 / 2\right), \\
\langle H\rangle_{\text {th }}^{2} & =(\hbar \omega)^{2}(\bar{n}+1 / 2)^{2} .
\end{aligned}
$$

The classical part of the entropy production can be calculated from the operator $D=\sum_{k} \delta_{k}\left(\tilde{Q}_{k}^{(1)}-Q_{k}^{(1)}\right)$ as

$$
\begin{aligned}
\operatorname{var}(D)= & \left\langle D^{2}\right\rangle_{\pi^{(1)}}=2 \sin ^{2}(g \tau)\left\{\delta \lambda_{1}^{2}\left[\cosh ^{2}(2 r)\left(\bar{n}^{2}+\bar{n}\right)+\sinh ^{2}(2 r)\left(\bar{n}^{2}+\frac{\bar{n}}{2}+\frac{1}{2}\right)\right]\right. \\
& +2 \delta \lambda_{1} \delta \lambda_{2} \cosh ^{2}(2 r) \sinh ^{2}(2 r)\left[\left(\bar{n}^{2}+\bar{n}\right)+\left(\bar{n}^{2}+\frac{\bar{n}}{2}+\frac{1}{2}\right)\right] \\
& \left.+\delta \lambda_{2}^{2}\left[\sinh ^{2}(2 r)\left(\bar{n}^{2}+\bar{n}\right)+\cosh ^{2}(2 r)\left(\bar{n}^{2}+\frac{\bar{n}}{2}+\frac{1}{2}\right)\right]\right\},
\end{aligned}
$$

where we have used the expressions obtained above in Eqs. (E2) and relations (E4) and (E5). The relative entropy reduction $\mathcal{R}$ in Eq. (21) is then obtained by substituting the above expression and the entropy production rate $\Sigma$ [Eq. (20)] into the definition of $\mathcal{R}$ in Eq. (14). 


\section{APPENDIX F: ONSAGER COEFFICIENTS FOR HEAT AND SQUEEZING}

In order to calculate the Onsager coefficients for the currents of heat and squeezing asymmetry, we proceed as in the previous subsection using expression (16). However, we now identify as charges $Q_{1}^{(1)}=H^{(1)}-\mu A^{(1)}$ and $Q_{2}^{(1)}=Q_{2}^{(1)}=A^{(1)}$, and affinity gradients $\delta \lambda_{1}^{\prime}=\delta \beta$ and $\lambda_{2}^{\prime}=-\beta \delta \mu$, leading to the reciprocity relations in Eqs. (22). In this case we obtain, for the SLDs,

$$
\begin{aligned}
\Lambda_{1}^{\prime} & =\langle H\rangle_{\pi^{(1)}}-\mu\langle A\rangle_{\pi^{(1)}}-H+\mu A, \\
\Lambda_{2}^{\prime} & =\langle A\rangle_{\pi^{(1)}}-\left(A+\left[\frac{\tanh (\alpha)}{\alpha}-1\right] \frac{1}{1-\mu^{2}}(A-\mu H)\right) \\
& =\Lambda_{2} .
\end{aligned}
$$

Notice that $\Lambda_{1}^{\prime}$ takes on the traditional Abelian form, since $\left[\Lambda_{1}^{\prime}, \pi_{\lambda}^{(1)}\right]=0$. Analogously, we obtain the following relevant operators:

$$
\begin{aligned}
Q_{1}^{(1)}-\tilde{Q}_{1}^{(1)}= & \sin ^{2}(g \tau)\left(H^{(1)}-\mu A^{(1)}\right) \\
& -\sin ^{2}(g \tau)\left(H^{(2)}-\mu A^{(2)}\right)-\cos (g \tau) \\
& \times \sin (g \tau) \omega\left[a_{1}^{\dagger} a_{2}+a_{1} a_{2}^{\dagger}\right. \\
& \left.+\mu\left(a_{1} a_{2}+a_{1}^{\dagger} a_{2}^{\dagger}\right)\right] \\
Q_{2}^{(1)}-\tilde{Q}_{2}^{\prime(1)}= & \sin ^{2}(g \tau) A^{(1)}-\sin ^{2}(g \tau) A^{(2)} \\
& -\cos (g \tau) \sin (g \tau) \omega\left(a_{1} a_{2}+a_{1}^{\dagger} a_{2}^{\dagger}\right) .
\end{aligned}
$$

Taking the trace over subsystem 2 in the above expressions and introducing them into Eq. (16) together with SLDs (F1), we obtain the set of coefficients reported in Eqs. (23) of Sec. V.

[1] H. B. Callen, Thermodynamics and an Introduction to Thermostatistics (Wiley, New York, 1985), 2nd ed.

[2] A. Sommerfeld and N. H. Frank, The statistical theory of thermoelectric, galvano- and thermomagnetic phenomena in metals, Rev. Mod. Phys. 3, 1 (1931).

[3] D. Beretta, N. Neophytou, J. M. Hodges, M. G. Kanatzidis, D. Narducci, M. Martin-Gonzalez, M. Beekman, B. Balke, G. Cerretti, W. Tremel, A. Zevalkink, A. I. Hofmann, C. Müller, B. Dörling, M. Campoy-Quiles, and M. Caironi, Thermoelectrics: From history, a window to the future, Mater. Sci. Eng.: R: Rep. 138, 100501 (2019).

[4] L. Onsager, Reciprocal relations in irreversible processes I, Phys. Rev. 37, 405 (1931).

[5] L. Onsager, Reciprocal relations in irreversible processes. II, Phys. Rev. 38, 2265 (1931).

[6] J. M. Horowitz and M. Esposito, Work producing reservoirs: Stochastic thermodynamics with generalized gibbs ensembles, Phys. Rev. E 94, 020102 (2016).

[7] R. Rao and M. Esposito, Conservation laws shape dissipation, New J. Phys. 20, 023007 (2018).
[8] J. A. Vaccaro and S. M. Barnett, Information erasure without an energy cost, Proc. R. Soc. A. 467, 1770 (2011).

[9] J. M. Hickey and S. Genway, Fluctuation theorems and the generalized gibbs ensemble in integrable systems, Phys. Rev. E 90, 022107 (2014).

[10] M. Perarnau-Llobet, A. Riera, R. Gallego, H. Wilming, and J. Eisert, Work and entropy production in generalised gibbs ensembles, New J. Phys. 18, 123035 (2016).

[11] Y. Guryanova, S. Popescu, A. J. Short, R. Silva, and P. Skrzypczyk, Thermodynamics of quantum systems with multiple conserved quantities, Nat. Commun. 7, 12049 (2016).

[12] N. Yunger Halpern and J. M. Renes, Beyond heat baths: Generalized resource theories for small-scale thermodynamics, Phys. Rev. E 93, 022126 (2016).

[13] J. Mur-Petit, A. Relaño, R. A. Molina, and D. Jaksch, Revealing missing charges with generalised quantum fluctuation relations, Nat. Commun. 9, 2006 (2018).

[14] N. Y. Halpern, Beyond heat baths II: Framework for generalized thermodynamic resource theories, J. Phys. A.: Math. Theor. 51, 094001 (2018).

[15] N. Yunger Halpern, M. E. Beverland, and A. Kalev, Noncommuting conserved charges in quantum many-body thermalization, Phys. Rev. E 101, 042117 (2020).

[16] P. Calabrese and J. Cardy, Evolution of entanglement entropy in one-dimensional systems, J. Stat. Mech.: Theory Exp. P04010 (2005).

[17] B. Bertini, F. Heidrich-Meisner, C. Karrasch, T. Prosen, R. Steinigeweg, and M. Žnidarič, Finite-temperature transport in one-dimensional quantum lattice models, Rev. Mod. Phys. 93, 025003 (2021).

[18] J. M. Bhat and A. Dhar, Transport in spinless superconducting wires, Phys. Rev. B 102, 224512 (2020).

[19] G. Manzano, The squeezed thermal reservoir as a generalized equilibrium reservoir, Phys. Rev. E 98, 042123 (2018).

[20] G. Manzano, F. Galve, R. Zambrini, and J. M. R. Parrondo, Entropy production and thermodynamic power of the squeezed thermal reservoir, Phys. Rev. E 93, 052120 (2016).

[21] N. Yunger Halpern, P. Faist, J. Oppenheim, and A. Winter, Microcanonical and resource-theoretic derivations of the thermal state of a quantum system with noncommuting charges, Nat. Commun. 7, 12051 (2016).

[22] M. Lostaglio, D. Jennings, and T. Rudolph, Thermodynamic resource theories, non-commutativity and maximum entropy principles, New J. Phys. 19, 043008 (2017).

[23] T. Croucher, S. Bedkihal, and J. A. Vaccaro, Discrete Fluctuations in Memory Erasure Without Energy Cost, Phys. Rev. Lett. 118, 060602 (2017).

[24] K. Fukai, Y. Nozawa, K. Kawahara, and T. N. Ikeda, Noncommutative generalized gibbs ensemble in isolated integrable quantum systems, Phys. Rev. Res. 2, 033403 (2020).

[25] I. Bloch, J. Dalibard, and W. Zwerger, Many-body physics with ultracold gases, Rev. Mod. Phys. 80, 885 (2008).

[26] J.-P. Brantut, C. Grenier, J. Meineke, D. Stadler, S. Krinner, C. Kollath, T. Esslinger, and A. Georges, A thermoelectric heat engine with ultracold atoms, Science 342, 713 (2013).

[27] M. Brunelli, L. Fusco, R. Landig, W. Wieczorek, J. Hoelscher-Obermaier, G. Landi, F. L. Semião, A. Ferraro, 
N. Kiesel, T. Donner, G. De Chiara, and M. Paternostro, Experimental Determination of Irreversible Entropy Production in Out-Of-Equilibrium Mesoscopic Quantum Systems, Phys. Rev. Lett. 121, 160604 (2018).

[28] M. T. Mitchison and P. P. Potts, in Thermodynamics in the Quantum Regime: Fundamental Aspects and New Directions, edited by F. Binder, L. A. Correa, C. Gogolin, J. Anders, and G. Adesso (Springer International Publishing, Cham, 2018), p. 149.

[29] K. Brandner, M. Bauer, and U. Seifert, Universal Coherence-Induced Power Losses of Quantum Heat Engines in Linear Response, Phys. Rev. Lett. 119, 170602 (2017).

[30] H. Kwon, H. Jeong, D. Jennings, B. Yadin, and M. S. Kim, Clock-Work Trade-Off Relation for Coherence in Quantum Thermodynamics, Phys. Rev. Lett. 120, 150602 (2018).

[31] Z. Holmes, S. Weidt, D. Jennings, J. Anders, and F. Mintert, Coherent fluctuation relations: From the abstract to the concrete, Quantum 3, 124 (2018).

[32] I. Henao and R. M. Serra, Role of quantum coherence in the thermodynamics of energy transfer, Phys. Rev. E 97, 062105 (2018).

[33] J. P. Santos, L. C. Céleri, G. T. Landi, and M. Paternostro, The role of quantum coherence in non-equilibrium entropy production, npj Quantum Inf. 5, 23 (2019).

[34] G. Francica, J. Goold, and F. Plastina, The role of coherence in the non-equilibrium thermodynamics of quantum systems, Phys. Rev. E 99, 042105 (2019).

[35] G. Manzano, R. Silva, and J. M. R. Parrondo, Autonomous thermal machine for amplification and control of energetic coherence, Phys. Rev. E 99, 042135 (2019).

[36] M. Lostaglio and M. P. Müller, Coherence and Asymmetry Cannot be Broadcast, Phys. Rev. Lett. 123, 020403 (2019).

[37] F. L. S. Rodrigues, G. De Chiara, M. Paternostro, and G. T. Landi, Thermodynamics of Weakly Coherent Collisional Models, Phys. Rev. Lett. 123, 140601 (2019).

[38] C. L. Latune, I. Sinayskiy, and F. Petruccione, Energetic and entropic effects of bath-induced coherences, Phys. Rev. A 99, 052105 (2019).

[39] K. Micadei, G. T. Landi, and E. Lutz, Quantum Fluctuation Theorems beyond Two-Point Measurements, Phys. Rev. Lett. 124, 090602 (2020).

[40] J. Rossnagel, O. Abah, F. Schmidt-Kaler, K. Singer, and E. Lutz, Nanoscale Heat Engine beyond the Carnot Limit, Phys. Rev. Lett. 112, 030602 (2014).

[41] J. Klaers, S. Faelt, A. Imamoglu, and E. Togan, Squeezed Thermal Reservoirs as a Resource for a Nano-Mechanical Engine beyond the Carnot Limit, Phys. Rev. X 7, 031044 (2017).

[42] G. Manzano, Entropy production and fluctuations in a Maxwell's refrigerator with squeezing, Eur. Phys. J. Spec. Top. 227, 285 (2018).

[43] J. Klaers, Landauer's Erasure Principle in a Squeezed Thermal Memory, Phys. Rev. Lett. 122, 040602 (2019).

[44] M. O. Scully, M. S. Zubairy, G. S. Agarwal, and H. Walther, Extracting work from a single heat bath via vanishing quantum coherence, Science 299, 862 (2003).

[45] O. Abah and E. Lutz, Efficiency of heat engines coupled to nonequilibrium reservoirs, EPL (Europhys. Lett.) 106, 20001 (2014)
[46] W. Niedenzu, V. Mukherjee, A. Ghosh, A. G. Kofman, and G. Kurizki, Quantum engine efficiency bound beyond the second law of thermodynamics, Nat. Commun. 9, 165 (2018).

[47] M. Esposito, K. Lindenberg, and C. Van den Broeck, Universality of Efficiency at Maximum Power, Phys. Rev. Lett. 102, 130602 (2009).

[48] R. Kubo, M. Toda, and N. Hashitsume, Statistical Physics II - Nonequilibrium (Springer-Verlag, Berlin, 1985) p. 279.

[49] D. Petz, Covariance and fisher information in quantum mechanics, J. Phys. A: Math. Gen. 35, 929 (2002).

[50] H. J. D. Miller, M. Scandi, J. Anders, and M. PerarnauLlobet, Work Fluctuations in Slow Processes: Quantum Signatures and Optimal Control, Phys. Rev. Lett. 123, 230603 (2019).

[51] M. Scandi, H. J. D. Miller, J. Anders, and M. PerarnauLlobet, Quantum work statistics close to equilibrium, Phys. Rev. Res. 2, 023377 (2020).

[52] H. J. D. Miller, M. H. Mohammady, M. Perarnau-Llobet, and G. Guarnieri, Thermodynamic Uncertainty Relation in Slowly Driven Quantum Heat Engines, Phys. Rev. Lett. 126, 210603 (2021).

[53] E. T. Jaynes, Information theory and statistical mechanics, Phys. Rev. 106, 620 (1957).

[54] T. Langen, S. Erne, R. Geiger, B. Rauer, T. Schweigler, M. Kuhnert, W. Rohringer, I. E. Mazets, T. Gasenzer, and J. Schmiedmayer, Experimental observation of a generalized gibbs ensemble, Science 348, 207 (2015).

[55] M. Rigol, V. Dunjko, V. Yurovsky, and M. Olshanii, Relaxation in a Completely Integrable Many-Body Quantum System: An Ab Initio Study of the Dynamics of the Highly Excited States of 1D Lattice Hard-Core Bosons, Phys. Rev. Lett. 98, 050405 (2007).

[56] J. Rau, Relaxation phenomena in spin and harmonic oscillator systems, Phys. Rev. 129, 1880 (1963).

[57] V. Giovannetti and G. M. Palma, Master Equations for Correlated Quantum Channels, Phys. Rev. Lett. 108, 040401 (2012).

[58] F. Ciccarello, Collision models in quantum optics, Quantum Meas. Quantum Metrol. 4, 53 (2017).

[59] G. De Chiara, G. Landi, A. Hewgill, B. Reid, A. Ferraro, A. J. Roncaglia, and M. Antezza, Reconciliation of quantum local master equations with thermodynamics, New J. Phys. 20, 113024 (2018).

[60] M. Cattaneo, G. De Chiara, S. Maniscalco, R. Zambrini, and G. L. Giorgi, Collision Models can Efficiently Simulate any Multipartite Markovian Quantum Dynamics, Phys. Rev. Lett. 126, 130403 (2021).

[61] H. P. Breuer and F. Petruccione, The Theory of Open Quantum Systems (Oxford University Press, New York, 2002), p. 636 .

[62] This unitary operation represents the interaction between reservoirs, e.g., through an interface with Hamiltonian $H_{\text {int }}$, such that $U \simeq e^{-} i H_{\text {int }} \tau$, where $\tau$ is the collision time.

[63] F. G. S. L. Brandão, M. Horodecki, J. Oppenheim, J. M. Renes, and R. W. Spekkens, Resource Theory of Quantum States out of Thermal Equilibrium, Phys. Rev. Lett. 111, 250404 (2013). 
[64] M. Horodecki and J. Oppenheim, Fundamental limitations for quantum and nanoscale thermodynamics, Nat. Commun. 4, 2059 (2013).

[65] F. G. S. L. Brandão, M. Horodecki, N. H. Y. Ng, J. Oppenheim, and S. Wehner, The second laws of quantum thermodynamics, Proc. National Acad. Sci. 112, 3275 (2015).

[66] G. Manzano, J. M. Horowitz, and J. M. R. Parrondo, Quantum Fluctuation Theorems for Arbitrary Environments: Adiabatic and Non-Adiabatic Entropy Production, Phys. Rev. X 8, 031037 (2018).

[67] P. Strasberg, G. Schaller, T. Brandes, and M. Esposito, Quantum and Information Thermodynamics: A Unifying Framework Based on Repeated Interactions, Phys. Rev. X 7, 021003 (2017).

[68] M. Esposito, K. Lindenberg, and C. Van den Broeck, Entropy production as correlation between system and reservoir, New J. Phys. 12, 013013 (2010).

[69] H. B. G. Casimir, On onsager's principle of microscopic reversibility, Rev. Mod. Phys. 17, 343 (1945).

[70] I. Marvian and R. W. Spekkens, Extending noether's theorem by quantifying the asymmetry of quantum states, Nat. Commun. 5, 1 (2014).

[71] A. Serafini, Quantum Continuous Variables: A Primer of Theoretical Methods (CRC Press, Boca Raton, 2017).

[72] J.-M. Pirkkalainen, E. Damskägg, M. Brandt, F. Massel, and M. A. Sillanpää, Squeezing of Quantum Noise of Motion in a Micromechanical Resonator, Phys. Rev. Lett. 115, 243601 (2015).

[73] E. E. Wollman, C. U. Lei, A. J. Weinstein, J. Suh, A. Kronwald, F. Marquardt, A. A. Clerk, and K. C. Schwab, Quantum squeezing of motion in a mechanical resonator, Science 349, 952 (2015).

[74] B. Yurke, P. G. Kaminsky, R. E. Miller, E. A. Whittaker, A. D. Smith, A. H. Silver, and R. W. Simon, Observation of 4.2-K Equilibrium-Noise Squeezing via a Josephson-Parametric Amplifier, Phys. Rev. Lett. 60, 764 (1988).

[75] Z. Jiang, Quantum fisher information for states in exponential form, Phys. Rev. A 89, 032128 (2014).

[76] G. Benenti, G. Casati, K. Saito, and R. S. Whitney, Fundamental aspects of steady-state conversion of heat to work at the nanoscale, Phys. Rep. 694, 1 (2017).

[77] For example, a bipartite interface consisting of two twolevel systems weakly interacting through a hopping Hamiltonian. Since the two-level systems are unnable to accomodate second-order coherence in their density operators, the squeezing current would be effectively blocked.

[78] H. Thierschmann, R. Sánchez, B. Sothmann, F. Arnold, C. Heyn, W. Hansen, H. Buhmann, and L. W. Molenkamp, Three-terminal energy harvester with coupled quantum dots, Nat. Nanotechnol. 10, 854 (2015).

[79] G. Maslennikov, S. Ding, R. Hablutzel, J. Gan, A. Roulet, S. Nimmrichter, J. Dai, V. Scarani, and D. Matsukevich, Quantum absorption refrigerator with trapped ions, Nat. Commun. 10, 202 (2019).

[80] D. W. C. Brooks, T. Botter, S. Schreppler, T. P. Purdy, N. Brahms, and D. M. Stamper-Kurn, Non-classical light generated by quantum-noise-driven cavity optomechanics, Nature 488, 476 (2012).

[81] J. Léonard, A. Morales, P. Zupancic, T. Donner, and T. Esslinger, Monitoring and manipulating higgs and goldstone modes in a supersolid quantum gas, Science $\mathbf{3 5 8}$, 1415 (2017).

[82] F. Mazza, R. Bosisio, G. Benenti, V. Giovannetti, R. Fazio, and F. Taddei, Thermoelectric efficiency of three-terminal quantum thermal machines, New J. Phys. 16, 085001 (2014).

[83] K. Brandner, K. Saito, and U. Seifert, Strong Bounds on Onsager Coefficients and Efficiency for Three-Terminal Thermoelectric Transport in a Magnetic Field, Phys. Rev. Lett. 110, 070603 (2013).

[84] B. Sothmann, R. Sánchez, and A. N. Jordan, Thermoelectric energy harvesting with quantum dots, Nanotechnology 26, 032001 (2014).

[85] G. Manzano, R. Sánchez, R. Silva, G. Haack, J. B. Brask, N. Brunner, and P. P. Potts, Hybrid thermal machines: Generalized thermodynamic resources for multitasking, Phys. Rev. Res. 2, 043302 (2020).

[86] D. Andrieux, P. Gaspard, T. Monnai, and S. Tasaki, The fluctuation theorem for currents in open quantum systems, New J. Phys. 11, 043014 (2009).

[87] M. Campisi, P. Talkner, and P. Hänggi, Fluctuation Theorems for Continuously Monitored Quantum Fluxes, Phys. Rev. Lett. 105, 140601 (2010).

[88] D. Andrieux and P. Gaspard, Quantum Work Relations and Response Theory, Phys. Rev. Lett. 100, 230404 (2008).

[89] F. Haake, Quantum Signatures of Chaos, springer series in synergetics, 3rd. ed. ed. (Springer, Berlin, 2010).

[90] A. B. Dutta, N. Mukunda, and R. Simon, The real symplectic groups in QM and optics, Pramana-J. Phys. 45, 471 (1995). 